\title{
Catalytic hydrogenation reactors for the fine chemicals industries. Their design and operation
}

\author{
K.R. Westerterp*, E.J. Molga ${ }^{1}$, K.B. van Gelder ${ }^{2}$ \\ Chemical Reaction Engineering Laboratories, Faculty of Chemical Engineering, University of Twente, P.O. Box 217, 7500 AE Enschede, \\ The Netherlands
}

Received 9 January 1996; accepted 30 May 1996

\begin{abstract}
The design and operation of reactors for catalytic hydrogenation in the fine chemical industries are discussed. The requirements for a good multiproduct catalytic hydrogenation unit as well as the choice of the reactor type are considered. Packed bed bubble column reactors operated without hydrogen recycle are recommended as the best choice to obtain a flexible reactor with good selectivities.

The results of an experimental study of the catalytic hydrogenation of 2,4-dinitrotoluene in a miniplant installation are presented to prove that the maximum yield in such a reactor can be achieved without a hydrogen recycle and with a hydrogen supply somewhat higher than the stoichiometric amount. Some characteristic properties of the reactor system and the influence of the reactor pressure and the hydrogen supply ratio are elucidated. Copyright (C) 1996 Elsevier Science S.A.
\end{abstract}

Keywords: Catalytic hydrogenation reactor; Reactor design; Packed bed bubble column reactor

\section{Introduction}

In fine chemicals industries the so-called Béchamps reaction has been widely used for hydrogenation reactions. Ilere hydrogen is produced by the interaction of $\mathrm{Fe}$ or $\mathrm{Zn}$ powder and hydrochloric acid, producing very active hydrogen. This reaction is executed in the batch mode in the liquid phase. The reaction rate can be controlled by the addition rate of the metal powder and the reaction occurs at low temperatures. These properties have made it the most important hydrogenation tool for fine chemicals. Regrettably, large amounts of waste materials are coproduced after neutralization, such as iron hydroxide sludges and neutralization salts, both often contaminated with organic chemicals. The amounts of waste materials are often 5 to 20 times

\footnotetext{
* Corresponding author.

1 Permanent address: Chemical and Process Engineering Department, Warsaw Technical University, ul. Warynskiego 1, 00-645 Warsaw, Poland.

2 Permanent address: Dow Benelux N.V., PDCD, P.O. Box 48, 4530 AA Terneuzen, The Netherlands.
}

larger than the amount of the desired chemicals produced. This waste problem has necessitated environmental protection measures and therefore catalytic hydrogenation has come under consideration in the fine chemicals industrics. Catalytic hydrogenation, of course, for many years has been applied in bulk chemical processes in specially designed rcactors. For fine chemicals a reactor installation is normally used for many different chemicals and production scrics usually last only from a few days to a few weeks, so that in the same installation 10 to 30 different chemical rcactions are often executed in the course of a year. Production quantities are so small that the construction of a unit dedicated to a single product is not economically feasible. Therefore, multiproduct use is a conditio sine qua non, also for a catalytic hydrogenation unit for fine chemicals. In our laboratories, therefore, a large expcrimental and theoretical study has been carried out to determine the most economical method to change from Béchamps to catalytic hydrogenation reactors [1]. To this end a number of possible reactor types have been tested and, in this paper, we will report on our initial 
considerations in coming to a selection, discuss the arguments for and against certain solutions and the final choice made.

We will also report on experimental work to corroborate our selection, the continuously operated, packed bubble column reactor filled with a packed catalyst bed and with hydrogen feed rates slightly higher than the stoichiometrically required amount.

\section{Requirements for a good multiproduct catalytic hydrogenation reactor for fine chemicals}

For catalytic hydrogenations we are usually concerned with three phases: gaseous hydrogen, an often dissolved component to be hydrogenated in the liquid phase and a heterogeneous solid catalyst. Homogeneous catalysis is not very widespread. A number of good three-phase reactors are available in process industries. Therefore, we will restrict ourselves to the classical three-phase reactors which already have proven their value in bulk chemicals processes. However, for chemicals applications a number of special requirements have to be met, which will be discussed in detail below.

\subsection{Universal catalyst}

In a multiproduct unit a universal catalyst should be applied. Quick changing of catalyst is difficult. Slurry catalysts, of course, can be washed out and filtered off, but their removal is time consuming and, for expensive catalysts in particular, filtration with a high efficiency is difficult to achieve and slow. Filtration can be avoided if a stationary bed of catalyst particles is used; changing a fixed bed catalyst is also time consuming. Therefore, it is preferred to keep the catalyst in the reactor system. In order to avoid the replacement each time by different catalysts, one universal catalyst should preferably be used for all different hydrogenation reactions to be executed. Ni, Pt and Pd catalysts are all fairly universal and the choice of the most suitable catalyst depends on the production programme. We tested all three and selected Pd. Ni catalysts are cheaper, but our applications demanded higher temperatures and hydrogen pressures to achieve the same conversion rates as with Pd catalysts. In the selection of a universal catalyst possible deactivation must also be considered and methods developed to regenerate the catalyst in an economic way.

\subsection{A universal method of heat removal}

Hydrogenation reactions are usually very exothermic. Therefore, a large cooling area must be installed in the reactor per unit volume and agitation levels must also be high in order to obtain high heat transfer coefficients. This necessitates extensive and complicated cooling coils or externally located circulating coolers for batch equipment. Further mixing and/or recirculation may influence the selectivity in a process.

As the method to remove the reaction heat under all circumstances and at all rates, we opted for heat withdrawal by evaporation of a solvent. Many reactions are executed in dissolution or the reactants are volatile at reaction conditions.

\subsection{A wide range of temperatures and solvents must be handled}

For many different hydrogenation reactions various solvents with different properties have to be used, especially with respect to vapour pressures and boiling points. Each reaction requires its own temperature range and this demands great flexibility of a multiproduct reactor.

\subsection{Good selectivities}

Many hydrogenations are carried out to completion, but when only partial hydrogenation of a molecule is required, selectivity also plays a major role. For multicomponent reactions of the consecutive type it is known that the batch or plug flow reactor gives higher yields and selectivities than mixed reactors, so mixing should be suppressed. This is often more complicated if competing reactions are of different reaction orders, but in general mixing should be avoided as much as possible.

\subsection{Ease of operation also without much information on kinetics}

The determination of adequate and reliable kinetic data is laborious and time consuming. To test threephase catalytic reactors we used as a test reaction the hydrogenation of 2,4-dinitrotoluene (DNT) to intermediate and final products. Langmuir-Hinselwood expressions were developed for the five main reactions and it took several man years to obtain accurate rate equations valid over a wide range of temperatures and pressures [2-4]. Such a large input of man hours cannot be afforded in fine chemicals industries, where often only a few tonnes of product are made in a short period of time. Therefore, units must be well operated with the highest possible selectivities and yields without detailed information on kinetics.

\subsection{Ease of control}

For a wide range of process conditions the reactor must be reliably controlled and kept at the desired 
conditions under all circumstances avoiding runaways or other instabilities. The control system must be easy to understand and to handle, so that operators can dedicate their attention above all to the execution of the ever changing recipes for the production of desired chemicals.

\subsection{Process economics}

It is evident that the process economics must be sound. This demands much detailed attention to equipment selection and mechanical design, as well as to further process development and easy possibilities for modifications.

Hydrogen excess and recirculation is costly, the gas carries away evaporated liquid, which has to be condensed in condensors with low heat transfer coefficients due to the presence of gas; after that the gas must be recompressed and recycled to the reactor. Compression is an expensive unit operation and, moreover, in recirculation systems a purge is required to prevent accumulation of inert components: this inevitably leads to hydrogen losses. Short switch over times must be feasible to reduce losses to off-spec products to a minimum. For the same reason rapid start-up and shut down methods must be developed. This also leads to long on-stream times and a good productive use of the plant.

Also the catalyst life is extremely important. Change of catalyst batches should be avoided as much as possible. The replacement of a fixed catalyst bed is cumbersome and involves a loss of possible production time. Thus some kind of rapid in situ regeneration is to be preferred.

\section{Choice of reactor type and batch or continuous mode of operation}

In fine chemicals industries the batch reactor is used almost exclusively. For catalytic hydrogenations the batch reactor has a number of disadvantages where a slurry catalyst is used. The complicated cooling coils, dead spaces behind baffles, etc. are difficult to clean if the catalyst has to be removed from the vessel. After each batch the vessel has to be emptied, which also implies that the catalyst falls dry. It is known that in this period, in particular, all reactants and products, still contained in the pores of the catalyst, may rapidly deteriorate via unwanted side reactions and so deactivate the catalyst. Deactivation usually starts as soon as the catalyst is no longer protected by the solvent. As a production series for one product consists of a number of batches, the exposure of the catalyst to deactivation conditions is frequent.

Despite the experience with batch reactors it may be worthwhile to operate continuous reactors for fine chemicals also. Continuously operated reactors only require one start-up and one shut-down during the production series of one product. This increases the operating time efficiency and prevents the deactivation of dry catalysts; this implies that the reactor volume can be much smaller than for batch reactors. As to the reactor type for three-phase systems an agitated slurry tank reactor $[5,6]$ is not advisable, because of the good mixing characteristics. For consecutive reaction systems in particular, the yields of desired products and selectivities will be considerably lower than in plug flow type reactors. The cocurrent downfiow trickle flow reactor [7-10] approaches best the plug flow character at high flow rates. Unfortunately, for a good wetting of the catalyst particles, liquid velocities must be high so that residence times usually are limited and not higher than a few minutes, up to a maximum of about a quarter of an hour. Many reactions require much longer reaction times. For that reason the packed bubble column reactor remains; this reactor has been tested by us and its many features have been amply discussed [11-13]. In this reactor the liquid and the gas flow cocurrently upward through the packed catalyst bed. It exhibits axial dispersion in the liquid phase [13], but by choosing a high and slender column the influence of the axial dispersion can be largely suppressed: under normal operating conditions and with not too large catalyst particles several 'reaction units' per meter column height can be obtained. By changing the liquid flow rate any residence time for the liquid phase can be achieved: a residence time of several hours is not a problem. This implies that selectivities approaching those in a plug flow reactor can be expected in this type of reactor, as is desired. For the case of heat removal in the reactor by evaporation of the reactant or a solvent and condensing the vapours with a cooler-condensor in the exit vapour line, a wide range of operating temperatures can be chosen by setting the reactor pressure. For these reasons we feel the packed bubble column reactor filled with a stationary solid catalyst bed is the best suited reactor with a very high versatility for application in the fine chemicals industries.

\section{Operation of the continuous packed bubble column reactor}

A number of the previously mentioned points will now be further elaborated. For further information see [14].

\subsection{Start-up and shut-down}

The solvent, which is heated up via a feed preheater to a temperature at which the reaction starts, can be put in the reactor-and-the reactor can be completely filled with liquid. 
Following this the liquid reactant feed and the hydrogen gas are supplied to the reactor in the desired ratio. The reaction starts, the reaction mixture heats up and at a temperature somewhat below the boiling point of the solvent at the set reactor pressure, the evaporation will become so high that a stable operating point is reached. Solvent vapours are condensed and returned to the reactor and the liquid phase leaves the top of the catalyst bed via an overflow. In order to stop the reaction the catalyst bed is washed out with pure solvent and the catalyst is kept covered with liquid.

\subsection{Switch over to a new product}

If for the new product a different solvent is used, first the old solvent is washed out over the top of the catalyst bed by the new solvent and the solvent mixture sent to the solvent recovery system of the plant. Following this the same start-up procedure as before is followed and the reactor pressure is adjusted if necessary. The catalyst is kept wet under liquid all the time in order to prevent decomposition reactions or coke formation at the catalyst surface if exposed to gas and/or air.

\subsection{Catalyst life and reactivation}

We observed that for our reactions the catalyst was prone to deactivation if the catalyst was exposed to gas or air. Via polymerisation and condensation reactions tar and coke were formed. We could reactivate our catalyst bed by burning off coke and tar [11] with air diluted with steam or nitrogen. The bed had to be preheated to a temperature where tar and coke combustion started. This empirically determined temperature in our case was $130^{\circ} \mathrm{C}$. In [15] we have described how the oxygen content in the regeneration gas can be determined and where thermocouples in the catalyst bed must be placed to be sure that at least one thermocouple is always located in the zone with the highest temperature in the bed, where the combustion takes place.

\subsection{Ease of control}

Besides rate controllers for the gas and liquid feed flows and liquid level controllers, the most important instrument is the reactor pressure controller. By setting the pressure the maximum temperature is also set. The liquid will evaporate at a temperature somewhat below the boiling temperature of the solvent or reactants at the set pressure. At the boiling temperature itself no hydrogen can dissolve anymore in the liquid and so the reaction would automatically stop. This also ensures that a runaway can never occur: the temperature can never surpass the solvent boiling temperature. By ad- justing the pressure we also automatically adjust the temperature level in the reactor.

A large temperature range can be covered depending on the vapour pressure curve of the liquid reaction phase and on the maximum allowable pressure of the reactor vessel.

\subsection{Hydrogen excess and gas recirculation}

In [1] and [12], on the basis of the analysis of a mathematical model describing the packed bubble column reactor for catalytic hydrogenation applications, it was suggested to supply a hydrogen stream slightly larger than the stoichiometrically required amount to hydrogenate the feedstock to the desired intermediate product (in order to produce an intermediate product in a consecutive reactions system). It was suggested that in this way the highest yield would also be automatically obtained. The reaction stops as soon as almost all hydrogen supplied has been consumed. This implies that the feed rate is so low that the reaction stops before the top of the catalyst bed is reached; or, in other words, the catalyst bed is larger than required for the desired conversion at the feed rate set. The hydrogen stream in such a case must be diluted, e.g. with nitrogen, in order to carry the solvent vapours to the condensor. The same holds, of course, for a complete hydrogenation. This mode of operating a packed bubble column reactor has two great advantages.

(1) We do not use an excess of hydrogen. This implies that we do not need to recirculate the excess of hydrogen and that the very costly hydrogen recycle compressor can be avoided, resulting in important investment and energy savings.

(2) We do not need to know the reaction kinetics; we only need to know the stoichiometry. Of course, in preliminary laboratory tests a suitable solvent has to be found and also a minimum temperature level has to be determined at which sufficiently high reaction rates are obtained. In the upper part of the catalyst bed, where the reaction has stopped, no temperature increase takes place anymore. In order to increase the capacity of the reactor the gas and liquid flow rates can be raised simultaneously as long as the last two thermocouples in the upper part of the reactor indicate equal temperatures; that is, the reaction is complete before the end of the catalyst bed.

These suggestions, based on a study of the mathematical model, have not been verified experimentally. Therefore, an experimental program, as explained in the following part, has been executed. We present results of the hydrogenation of 2,4-DNT obtained in a miniplant packed bed bubble column reactor and in methanol as the evaporating solvent. The corresponding reaction network consisting of parallel and consecu- 


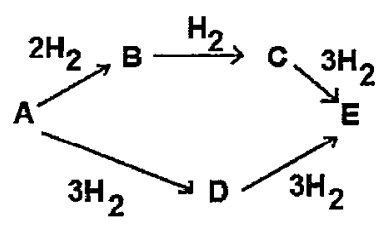

Fig. 1. Reaction scheme for the catalytic hydrogenation of 2,4-DNT.

tive pathways is shown in Fig. 1. The aim of our work is to determine the best method of operating the reactor to obtain the maximal yield of an intermediate product.

\section{Experimental installation and procedure}

The flow scheme of the miniplant installation is shown in Fig. 2. The reactor consists of a cylindrical stainless steel shell 11 and an internal tube 12 which contains the catalyst bed. The basic dimensions of the shell and the inner tubes used are given in Table 1. The length of the catalyst bed can be varied by using inner tubes of different heights as shown in Table 1. Both gas and liquid enter the reactor at the bottom and flow cocurrently to the top of the bed. The gas leaves the reactor at the top, whereas the liquid flows over the edge of the inner tube and downward in the annulus between the shell and the inner tube and then leaves the
Table 1

Basic dimensions of the reactor

\begin{tabular}{lll}
\hline Reactor shell: & length & $L_{\mathrm{s}}=1120 \mathrm{~mm}$ \\
& internal diameter & $D_{\mathrm{s}}=150 \mathrm{~mm}$ \\
Catalyst bed: & length & $L=970$ or $710 \mathrm{~mm}$ \\
& internal diameter & $D=120 \mathrm{~mm}$
\end{tabular}

Location of the thermocouple and sampling points (Fig. 2):

\begin{tabular}{lll}
\hline Location number & \multicolumn{2}{l}{ Height above the bed entrance } \\
\cline { 2 - 3 } & $L=970 \mathrm{~mm}$ & $L=710 \mathrm{~mm}$ \\
\hline top & 0.97 & 0.71 \\
1 & 0.75 & 0.60 \\
2 & 0.35 & 0.35 \\
3 & 0.13 & 0.12 \\
\hline
\end{tabular}

reactor at the bottom. A heating jacket is installed around the reactor shell to shorten the time required to reach steady state conditions and to maintain an almost adiabatic operation of the reactor. For further details and drawings see [11]. The liquid feed is stored in a jacketed supply vessel 13 equipped with a U-shaped agitator. This vessel is heated by means of hot water from a thermostat. Before starting an experiment oxygen is stripped out of the solution by slow bubbling through of nitrogen. The liquid is fed to the reactor by means of a Lewa membrane pump 14 and the flow is

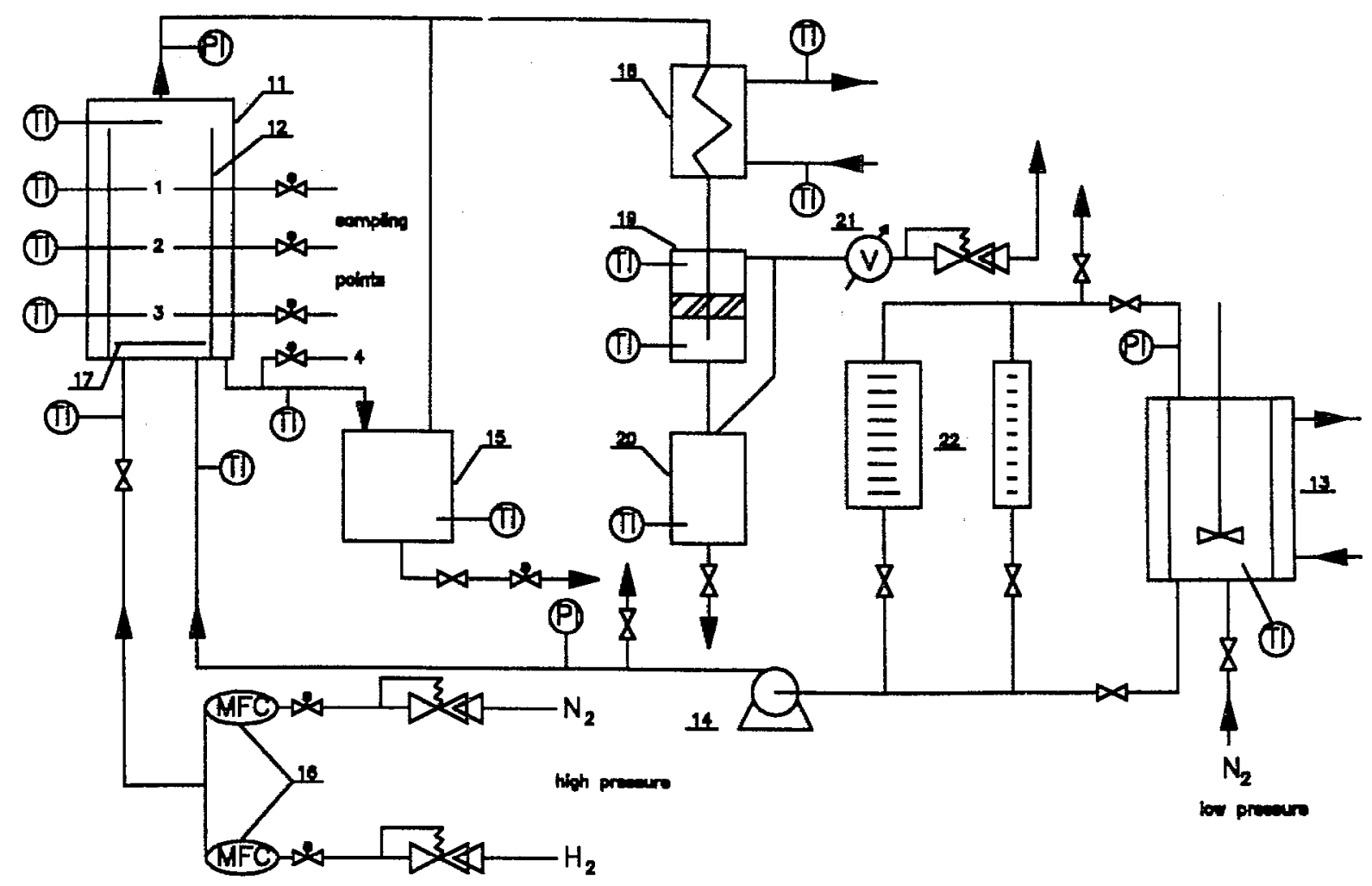

Fig. 2. Flowsheet of the miniplant installation. PI, pressure indicator; TI, temperature indicator; MFC, mass flow controller. See text for further details. 
controlled by changing the stroke length. By means of two burettes 22 in the liquid line the pump can be calibrated. The liquid reaction product is collected in a buffer vessel 15 . The gas leed, being either hydrogen or a hydrogen-nitrogen mixture, is taken from high pressure feed lines. Magnetic valves are installed in both feed lines and the flow rates of hydrogen and nitrogen are controlled by Brooks 581 Mass Flow Controllers 16.

Gas and liquid are mixed before entering the reactor bed in a gas-liquid chamber 17 at the inlet of the reactor. The gas leaving the reactor flows through a condenser 18 , where evaporated solvent is condensed, and then to the gas-liquid separator 19. The condensed solvent is collected in a separate buffer vessel 20 and returned to the reactor if necessary; the gas leaves the system through a backpressure valve controlling the pressure in the system. The volumetric flow of the effluent gas is measured with a gas meter 21 .

A system of sampling tubes and thermocouple tubes, inserted directly into the bed, enables us to determine the temperature as well as the liquid phase composition at four locations along the reactor. The sampling tubes are equipped with magnetic valves installed outside the reactor. The location of the sampling and thermocouple tubes is given in Table 1 .

The reactor system is automated with a computer HP 9816 and a data aquistion and control unit HP 3497A of Hewlett Packard. The programme a.o. checks if all variables are within preset safety limits. In case of emergency the programme switches the gas feed from $\mathrm{H}_{2}$ to $\mathrm{N}_{2}$ and stops the liquid feed. The key variables are the temperatures in the catalyst bed, the gas and the liquid inlet temperatures as well as the gas and the liquid outlet temperatures and further the reactor pressure, the inlet and the outlet gas flow rates and the liquid feed flow rate. They are temporarily stored in computer memory and later transferred to floppy disk. The temperatures at all other locations are monitored and recorded with a Philips PM 8237A multipoint data recorder.

The catalyst used is a shell catalyst, contains 0.08 wt.\% of $\mathrm{Pd}$ on $4 \times 4 \mathrm{~mm}$ porous cylindrical alumina pellets and is manufactured by Girdler Süd Chemie A.G. The penetration depth of palladium in the cylindrical support is estimated to be about $100 \mu \mathrm{m}$. Methanol is used as an evaporating solvent. A solution of DNT in methanol, usually in the range of 0.11 to $0.20 \mathrm{kmol} / \mathrm{m}^{3}$, is used as a feed.

About one hour after reaching steady-state conditions liquid samples are collected and immediately analysed with a Varian 3300 gas chromatograph using a $6 \mathrm{ft} \times 8^{\prime \prime}$ Tenax 60/80 mesh column and a HP 3392A integrator. The entire sampling and analyzing sequence at all detection points takes between 2 and $3 \mathrm{~h}$, during which period all operating parameters are kept con- stant. The whole experiment, including warming up of the reactor, stabilizing conditions, sampling procedure and cooling down of the reactor, usually takes about 7-8 h. Temperatures measured in the reactor bed during the entire experiment are plotted in Fig. 3 for Run 4. We can see that steady state is reached after $1.5-2 \mathrm{~h}$ from the start of the process, which is equivalent to 4-5 times the liquid residence time. At a hydrogen supply ratio - defined as molar hydrogen feed rate over the stoichiometric molar rate needed to convert all DNT to DAT - of $\alpha_{\text {DAT }}<1$ (Runs 13-22) equilibration of the reactor usually took as much as 2 to $3 \mathrm{~h}$.

\section{Experimental results}

To describe the reactor performance the following aspects have been taken into account:

(1) The pressure drop is small in comparison to the reactor pressure: the reactor is practically isobaric.

(2) Because of the heating jacket temperature following the reactor temperature we can also assume that the reactor is nearly adiabatic.

(3) The temperatures of the gas and the liquid phases are equal to each other over the cross-section of the reactor bed.

(4) The flow regime in our reactor was checked with criteria given in the literature. The gas mass velocity was never higher than $2.5 \times 10^{-3} \mathrm{~kg} \mathrm{~m}^{-2} \mathrm{~s}^{-1}$ and the mass flow ratio $\rho_{\mathrm{L}} u_{\mathrm{L}} /\left(\rho_{\mathrm{G}} u_{\mathrm{G}}\right)$ varied between 10 and 35 ; therefore, according to the flow map by Turpin and Huntington [16], all our experiments have been performed in the bubble flow regime. With a minimum gas velocity of $u_{\mathrm{G}, \min }=0.3 \times$ $10^{-3} \mathrm{~m} \mathrm{~s}^{-1}$ according to the criterion of Saada [17] two-phase flow must take place in all voids for all operating conditions. So the available catalyst area is fully wetted and utilized.

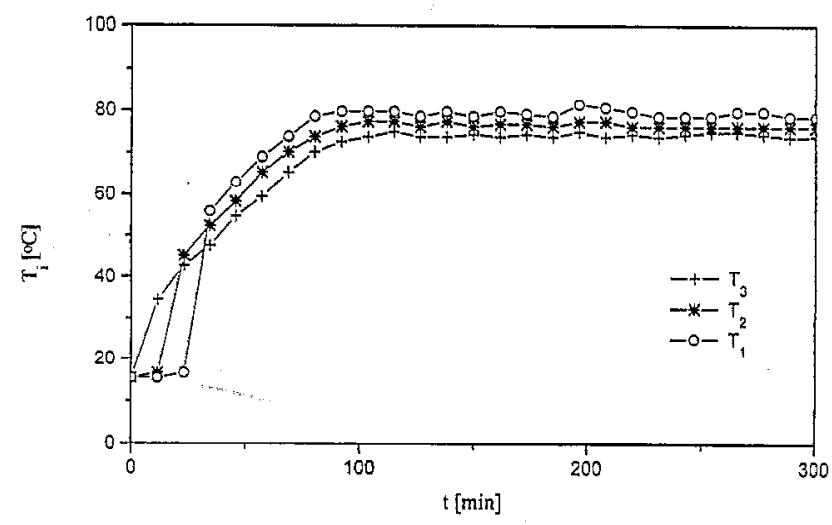

Fig. 3. Measured reactor bed temperatures-Run 4. Locations of thermocouples: 3-points $(+)$, 2-points (*), 1-point (O) - see Fig. 2. 
Table 2

Experimental conditions and results

\begin{tabular}{|c|c|c|c|c|c|c|c|c|c|c|}
\hline Run & $\begin{array}{l}P \\
(\mathrm{MPa})\end{array}$ & $\begin{array}{l}q_{\mathrm{L} \text { in }} \times 10^{6} \\
\left(\mathrm{~m}^{3} \mathrm{~s}^{-1}\right)\end{array}$ & $\begin{array}{l}q_{\mathrm{H} 2 \text { in }} \times 10^{6} \\
\left(\mathrm{~N} \mathrm{~m}^{3} \mathrm{~s}^{-1}\right)\end{array}$ & $\begin{array}{l}q_{\mathrm{N} 2 \text { in }} \times 10^{6} \\
\left(\mathrm{Nm}^{3} \mathrm{~s}^{-1}\right)\end{array}$ & $\begin{array}{l}q \mathrm{~g} \text { out } \times 10^{6} \\
\left(\mathrm{~N} \mathrm{~m}^{3} \mathrm{~s}^{-1}\right)\end{array}$ & $\begin{array}{l}\alpha_{\mathrm{DAT}} \\
(-)\end{array}$ & $\begin{array}{l}T_{\circ} \\
\left({ }^{\circ} \mathrm{C}\right)\end{array}$ & $\begin{array}{l}T_{\mathrm{O} \text { in }} \\
\left({ }^{\circ} \mathrm{C}\right)\end{array}$ & $\begin{array}{l}L \\
(\mathrm{~m})\end{array}$ & $\begin{array}{l}c_{\mathrm{Ao}} \\
\left(\mathrm{mol} \mathrm{m}{ }^{-3}\right)\end{array}$ \\
\hline 1 & 0.49 & 2.78 & 258.3 & 0.0 & 208.3 & 4.9 & 50 & 43.5 & 0.97 & 140 \\
\hline 2 & 0.50 & 2.72 & 138.3 & 0.0 & 91.7 & 2.7 & 50 & 46.1 & 0.97 & 140 \\
\hline 3 & 0.22 & 2.83 & 138.3 & 0.0 & 110.0 & 2.6 & 50 & 42.7 & 0.97 & 180 \\
\hline \multicolumn{11}{|c|}{ Fresh catalyst charge } \\
\hline 4 & 0.30 & 2.83 & 98.3 & 0.0 & 76.7 & 2.3 & 35 & 32.9 & 0.71 & 110 \\
\hline 5 & 0.28 & 2.78 & 125.0 & 0.0 & 115.0 & 1.9 & 35 & 32.2 & 0.71 & 180 \\
\hline 6 & 0.46 & 2.78 & 125.0 & 0.0 & 115.0 & 1.9 & 35 & 33.2 & 0.71 & 180 \\
\hline 7 & 0.50 & 2.78 & 96.7 & 0.0 & 50.0 & 1.2 & 50 & 47.2 & 0.71 & 210 \\
\hline 8 & 0.41 & 2.89 & 66.7 & 0.0 & 46.7 & 1.5 & 45 & 43.2 & 0.71 & 110 \\
\hline 9 & 0.30 & 2.97 & 75.0 & 0.0 & 55.0 & 1.6 & 45 & 42.4 & 0.71 & 120 \\
\hline 10 & 0.59 & 2.25 & 60.0 & 0.0 & 46.7 & 1.7 & 4.5 & 43.5 & 0.71 & 120 \\
\hline 11 & 0.40 & 2.64 & 166.7 & 0.0 & 140.0 & 4.0 & 45 & 40.5 & 0.71 & 120 \\
\hline 12 & 0.41 & 2.39 & 36.7 & 0.5 & 18.3 & 0.6 & 50 & 48.1 & 0.71 & 180 \\
\hline \multicolumn{11}{|c|}{ Catalyst activity stabilized } \\
\hline 13 & 0.40 & 2.72 & 45.0 & 10.0 & 20.0 & 0.6 & 50 & 47.9 & 0.71 & 190 \\
\hline 14 & 0.40 & 2.75 & 45.0 & 10.0 & 20.0 & 0.6 & 45 & 43.4 & 0.71 & 190 \\
\hline 15 & 0.41 & 2.72 & 45.0 & 20.0 & 31.7 & 0.6 & 45 & 43.1 & 0.71 & 190 \\
\hline 16 & 0.40 & 1.36 & 21.7 & 5.0 & 6.7 & 0.65 & 45 & 43.4 & 0.71 & 180 \\
\hline 17 & 0.20 & 1.39 & 21.7 & 5.0 & 12.5 & 0.62 & 45 & 42.0 & 0.71 & 190 \\
\hline 18 & 0.81 & 1.39 & 21.7 & 5.0 & 6.0 & 0.62 & 45 & 44.2 & 0.71 & 200 \\
\hline 19 & 0.81 & 1.39 & 26.7 & 5.8 & 9.3 & 0.72 & 45 & 44.0 & 0.71 & 200 \\
\hline 20 & 0.81 & 1.39 & 18.3 & 4.2 & 4.2 & 0.52 & 45 & 44.3 & 0.71 & 190 \\
\hline 21 & 0.81 & 1.36 & 14.0 & 3.7 & 4.3 & 0.42 & 45 & 44.4 & 0.71 & 180 \\
\hline 22 & 0.41 & 2.72 & 45.0 & 10.0 & 18.3 & 0.6 & 50 & 48.0 & 0.71 & 180 \\
\hline Run & $\begin{array}{l}T_{3} \\
\left({ }^{\circ} \mathrm{C}\right)\end{array}$ & $\begin{array}{l}T_{2} \\
\left({ }^{\circ} \mathrm{C}\right)\end{array}$ & $\begin{array}{l}T_{1} \\
\left({ }^{\circ} \mathrm{C}\right)\end{array}$ & $\begin{array}{l}\xi \\
(-)\end{array}$ & $\begin{array}{l}\xi \\
(-)\end{array}$ & $\begin{array}{l}\xi \\
(-)\end{array}$ & $\begin{array}{l}\xi \\
(-)\end{array}$ & $\begin{array}{l}\xi \\
(-)\end{array}$ & & \\
\hline 1 & 72 & 86 & 87 & 0.87 & 0.92 & 0.94 & 0.95 & 1.03 & & \\
\hline 2 & 96 & 100 & 100 & 0.91 & 0.97 & 0.98 & 0.98 & 0.96 & & \\
\hline 3 & 59 & 62 & 65 & 0.35 & 0.44 & 0.55 & 0.57 & 0.53 & & \\
\hline \multicolumn{11}{|c|}{ Fresh catalyst charge } \\
\hline 4 & 74 & 76 & 79 & - & - & 0.47 & 0.48 & 0.56 & & \\
\hline 5 & 36 & 36 & 37 & 0.13 & 0.14 & 0.14 & 0.15 & 0.18 & & \\
\hline 6 & 39 & 38 & 38 & 0.15 & 0.16 & 0.16 & 0.16 & 0.18 & & \\
\hline 7 & 74 & 88 & 91 & 0.52 & 0.54 & 0.56 & 0.58 & 0.61 & & \\
\hline 8 & 74 & 77 & 80 & 0.41 & 0.43 & 0.49 & 0.48 & 0.50 & & \\
\hline 9 & 64 & 64 & 65 & 0.30 & 0.34 & 0.40 & 0.40 & 0.44 & & \\
\hline 10 & 72 & 75 & 77 & 0.35 & 0.42 & 0.51 & 0.52 & 0.45 & & \\
\hline 11 & 72 & 73 & 74 & 0.48 & 0.51 & 0.50 & 0.55 & 0.57 & & \\
\hline 12 & 77 & 80 & 81 & 0.40 & 0.44 & 0.45 & 0.46 & 0.48 & & \\
\hline \multicolumn{11}{|c|}{ Catalyst activity stabilized } \\
\hline 13 & 89 & 90 & 92 & 0.52 & 0.54 & 0.55 & 0.55 & 0.54 & & \\
\hline 14 & 86 & 89 & 92 & 0.47 & 0.49 & 0.50 & 0.52 & 0.51 & & \\
\hline 15 & 87 & 88 & 90 & 0.47 & 0.47 & 0.48 & 0.50 & 0.51 & & \\
\hline 16 & 93 & 93 & 95 & 0.50 & 0.51 & 0.52 & 0.53 & 0.53 & & \\
\hline 17 & 72 & 72 & 72 & 0.43 & 0.43 & 0.43 & 0.43 & 0.41 & & \\
\hline 18 & 107 & 110 & 113 & 0.60 & 0.61 & 0.61 & 0.61 & 0.62 & & \\
\hline 19 & 116 & 118 & 120 & 0.62 & 0.62 & 0.63 & 0.63 & 0.62 & & \\
\hline 20 & 101 & 105 & 107 & 0.50 & 0.51 & 0.52 & 0.54 & 0.52 & & \\
\hline 21 & 83 & 90 & 92 & 0.12 & 0.45 & 0.44 & 0.44 & 0.40 & & \\
\hline 22 & 89 & 92 & 94 & 0.49 & 0.53 & 0.54 & 0.55 & 0.56 & & \\
\hline
\end{tabular}

The most important experimental data are given in Table 2. The hydrogen conversion has been defined as the ratio of the amount of hydrogen actually consumed to the amount of hydrogen consumed for a complete conversion of DNT to DAT. According to this definition experimental values of the hydrogen conversion at the $j$-th measurement point have been determined as follows: 
$\zeta_{j}=\frac{1}{3} X_{\mathrm{B}}+\frac{1}{2}\left(X_{\mathrm{C}}+X_{\mathrm{D}}\right)+X_{\mathrm{E}}$

where $X_{i}$ are relative molar fractions of the $i$-th reactant in the liquid phase, respectively, defined as follows:

$$
X_{i}=\frac{x_{i}}{\sum x_{i}}
$$

The temperature of samples taken at different locations in the packed bed was sometimes as high as $393 \mathrm{~K}$, so a certain amount of methanol vapour was flashed off and consequently the measured molar concentrations of the organic compounds were higher than in the reactor. On the basis of Eqs. (1) and (2) we still obtain the correct hydrogen conversions, automatically compensated for by the evaporation loss during sampling.

The overall hydrogen conversion $\zeta_{\text {top,cal }}$ as measured at the top of the catalyst bed can also be calculated according to the equation:

$\zeta_{\text {top }, \text { cal }}=\frac{\varphi_{\mathrm{II0}}-\varphi_{\mathrm{H}, \mathrm{out}}}{6 q_{\mathrm{L} 0} c_{\mathrm{A} 0}}$

where $\varphi_{\mathrm{H} 0}$ and $\varphi_{\mathrm{H}, \text { out }}$ are the inlet and the outlet molar hydrogen flow rates and $q_{\mathrm{L}}$ the inlet flow rate of the liquid. The hydrogen supply ratio $\alpha_{\text {DAT }}$ is defined as:

$\alpha_{\mathrm{DAT}}=\frac{\varphi_{\mathrm{H} 0}}{6 q_{\mathrm{L} 0} c_{\mathrm{A} 0}}$

The hydrogen conversions determined from concentration measurements, $\zeta_{\text {top }}$ (Eq. (1)) compared well with those calculated with Eq. (3), $\zeta_{\text {top,cal }}$, as given in Table 2 ; the differences exceeded $10 \%$ only for a few experiments.

In Table 2 the amount of gas measured in the outlet is given. It mainly consists of nitrogen, non-converled hydrogen and non-condensed methanol vapour. The amount of methanol vapour condensed was measured and found to vary between 1.3 and $127 \times 10^{-6} \mathrm{~N} \mathrm{~m}^{3}$ $\mathrm{s}^{-1}$.

\subsection{Deactivation of the catalyst}

In experiment 4 the catalyst was fresh, while experiment 5 was carried out 2 days later with the same catalyst. We can observe in Table 2 that deactivation of the catalyst is very fast. The decrease in the catalyst activity becomes smaller after several weeks. As catalyst deactivation may be caused by an irreversible adsorption of reaction products we washed the catalyst bed after every experiment with methanol with a volume five times the liquid hold-up of the reactor; in between the two experiments we kept the reactor filled with methanol and under nitrogen. Comparing Runs 13 and 22 , which were executed with the same catalyst, we see that the activity can be stabilized in this way and that deactivation does not occur any more. Run 22 was executed 58 days after Run 13. Therefore, in the main series of experiments of Runs 13-22, performed to investigate the influence of the hydrogen supply ratio and the reactor pressure under conditions where $\alpha_{\text {DAT }}<1$, we are sure that the catalyst activity is almost constant and reproducible.

\subsection{Reactor operation}

Observing the dependence of the total hydrogen conversion and the reactor pressure in Fig. 4 at $P=0.8$ $\mathrm{MPa}$ only it can be seen that almost all supplied hydrogen is consumed. At lower hydrogen partial pressure, the reaction rate significantly decreases, so not all supplied hydrogen can be converted in the reactor: the total hydrogen conversion $\zeta_{\text {top }}$ is smaller than the hydrogen supply ratio $\alpha_{\text {DAT }}$. For a hydrogen supply ratio of $\alpha_{\mathrm{DAT}}<1$ and at long enough residence times we can expect the total hydrogen conversion $\zeta_{\text {top }}$ to equal $\alpha_{\mathrm{DAT}}$ since all supplied hydrogen is converted.

The data in Table 2 show that for the Runs 13 to 22 the amount of nitrogen as sweep gas added to the hydrogen feed was $18-19 \%$. For Run 15 it was $31 \%$ and for Run 21 it was $21 \%$. We further observe from Table 2 that the nitrogen content in the off-gases varies from 41 to almost $100 \%$. It is evident that this strongly influences the partial pressure of the hydrogen in the major part of the reactor. As this outlet concentration of hydrogen is not a variable that can be set a priori, we have not studied its influence further and kept the nitrogen inlet concentrations at $18-19 \%$.

\subsection{Yield of the intermediate product $C$}

The optimization of the production of intermediates without an expensive recycle of recompressed hydrogen, can be achieved at a hydrogen supply ratio $\alpha_{\mathrm{DAT}}<1$.

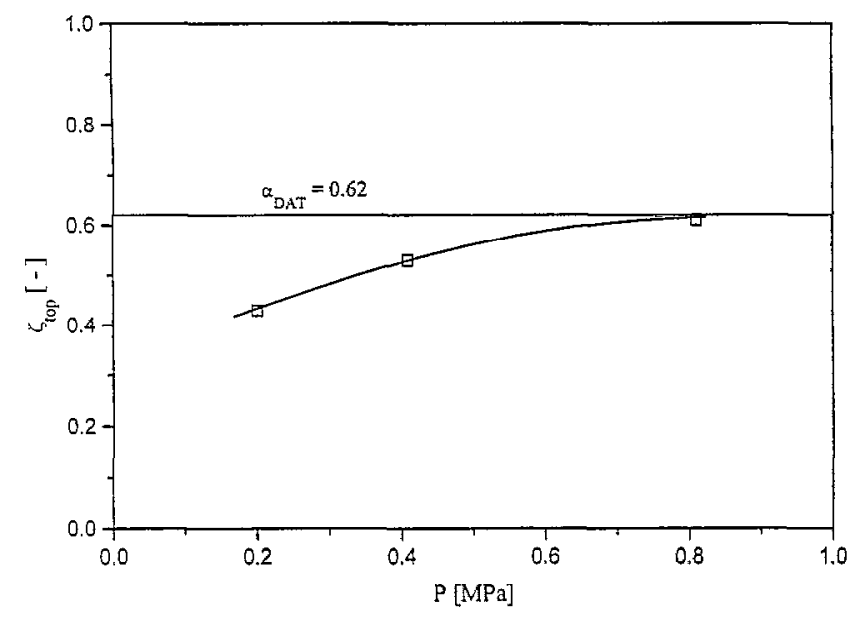

Fig. 4. Hydrogen conversions as a function of reactor pressure $P$. Fixed hydrogen supply ratio $\alpha_{\mathrm{DAT}}=0.62$. 


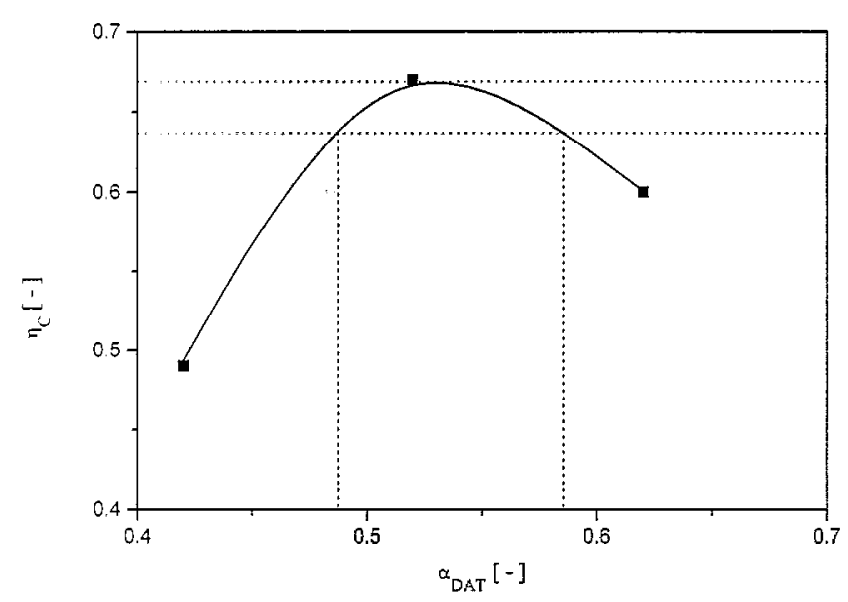

Fig. 5. Yield of the intermediate $\mathrm{C}$ at the reactor outlet as a function of the hydrogen supply ratio $\alpha_{\mathrm{DAT}}$. Fixed reactor pressure $P=0.81$ MPa.

The yield of the intermediate product $\mathrm{C}, \eta_{\mathrm{C}}$, can be determined directly from the concentration measurements because $\eta_{\mathrm{C}}=X_{\mathrm{C}}$. For economical reasons it is also important that all supplied hydrogen is converted. The influence of the hydrogen supply ratio on the yield towards the intermediate compound $\mathrm{C}$ at $P=0.81 \mathrm{MPa}$ is shown in Fig. 5. A distinct maximum in the yield of component $\mathrm{C}$ is noticed in this figure at a fixed value of the hydrogen supply ratio. At $\alpha_{\mathrm{DAT}}=0.52$ and at $P=$ $0.81 \mathrm{MPa}$ the highest value of $\eta_{\mathrm{C}}=0.67$ is obtained, and almost all supplied hydrogen is converted at these conditions. In Table 3 all yields $\eta_{C}$ as obtaincd arc given and in Table 4 data for the composition of the reaction mixture at the various locations in the reactor bed are given for a selected run.

In Fig. 5 we have indicated with a full line the yields as calculated with the model discussed in [12] and with the kinetics given in [4]; the range is indicated with a band, where the yield deviates less than five per cent of the maximum yield. It demonstrates that by setting a stoichiometric hydrogen supply ratio of a little above 0.50 the optimum yield is approached almost exactly.

Table 3

Experimental yield of the intermediate product $\mathrm{C}$

\begin{tabular}{lllll}
\hline Run & $\begin{array}{l}P \\
(\mathrm{MPa})\end{array}$ & $\begin{array}{l}\alpha_{\text {DAT }} \\
(-)\end{array}$ & $\begin{array}{l}\xi_{\text {top }} \\
(-)\end{array}$ & $\begin{array}{l}\eta_{\mathrm{C}} \\
(-)\end{array}$ \\
\hline 13 & 0.4 & 0.60 & 0.55 & 0.58 \\
14 & 0.4 & 0.60 & 0.52 & 0.55 \\
15 & 0.4 & 0.60 & 0.50 & 0.59 \\
16 & 0.4 & 0.65 & 0.53 & 0.63 \\
17 & 0.2 & 0.62 & 0.43 & 0.48 \\
18 & 0.81 & 0.62 & 0.61 & 0.60 \\
19 & 0.81 & 0.72 & 0.63 & 0.59 \\
20 & 0.81 & 0.52 & 0.54 & 0.66 \\
21 & 0.81 & 0.42 & 0.44 & 0.49 \\
22 & 0.81 & 0.60 & 0.55 & 0.63 \\
\hline
\end{tabular}

Table 4

Typical reactant concentrations along the reactor bed - Run 20

\begin{tabular}{llllll}
\hline Location & $x_{\mathrm{A}}$ & $x_{\mathrm{B}}$ & $x_{\mathrm{C}}$ & $x_{\mathrm{D}}$ & $x_{\mathrm{E}}$ \\
\hline 3 & 0.07 & 0.00 & 0.66 & 0.20 & 0.07 \\
2 & 0.04 & 0.01 & 0.67 & 0.21 & 0.07 \\
1 & 0.02 & 0.00 & 0.69 & 0.22 & 0.07 \\
Top & 0.01 & 0.00 & 0.67 & 0.23 & 0.09 \\
\hline
\end{tabular}

For consecutive reactions, of course, it is impossible to know what the correct ratio is without knowing the kinetics. In our case of the hydrogenation of 2,4-DNT we can only be sure that it must be beyond $\alpha=0.50$ because, at maximum yield, part of $\mathrm{C}$ has already been converted into $\mathrm{E}$. Therefore, in practice the operator would set the ratio, e.g. to 0.55 , and then empirically increase it slowly step by step until the maximum yield is cventually rcached. In our casc, with ratios between 0.49 and 0.59 , we lose less than $5 \%$ of our raw material, which must be acceptable in the initial period of a run. We also have seen that a certain minimum pressure is required. This also has to be determined step by step; in our case pressures of 0.2 and $0.4 \mathrm{MPa}$, see Fig. 4 , are still somewhat too low; at $0.8 \mathrm{MPa}$ the reactor runs fine.

\section{Summary and conclusions}

Experimental results obtained in a miniplant installation with a packed bed bubble column reactor for the catalytic hydrogenation of 2,4-DNT with an evaporating solvent have been presented and discussed. A deactivation of the catalyst has been noticed and a method to stabilize the catalyst activity by a special treatment of the bed after each experimental run has been developed. The influence of operating parameters, such as the hydrogen supply ratio and the pressure of the reactor, has been discussed.

The method, as suggested by Westerterp et al. [1] and by van Gelder et al. [12], to obtain the maximal yield of an intermediate product without hydrogen recycle has been experimentally evaluated. The packed bubble column reactor can be run with a correct amount of hydrogen supplied to achieve the maximum yield of intermediate product at a certain feed rate of reactant in the liquid phase. A small amount of the inert gas in our case nitrogen - must be added to the reactor to ensure a stable performance of the reactor when almost all supplied hydrogen is consumed. The reactor pressure must be high enough to maintain a sufficiently high hydrogen consumption rate and ensure almost complete conversion. Both the hydrogen supply ratio, as well as the required reactor pressure, can be determined experimentally without studying reaction kinet- 
ics. For the investigated system, after stabilizing the catalyst activity, good conditions were $\alpha_{\mathrm{DAT}}=0.53$ and $P=0.81 \mathrm{MPa}$ at which the maximal yield of the intermediate product $C$ is obtained without loss of un-consumed hydrogen. This proves the suggestion of Westerterp et al. [1] and Van Gelder et al. [12] that the maximum yield of a desired product can be obtained with a supply of hydrogen slightly higher than the stoichiometric value, provided the reactor pressure is set high enough for sufficiently high reaction rates to convert the hydrogen supplied.

We also discussed the choice of the reactor. A batch reactor has a much larger volume per unit of reaction product and tank-like pressure vessels are much more expensive than cylindrical vessels. This, combined with the difficulties of handling catalyst slurries and above all of preventing losses of the often rather expensive catalysts, made us consider continuously operating reactors with fixed catalyst beds also. We eventually selected the packed bubble column as a suitable reactor.

We should also mention some drawbacks of the proposed reactor. Axial mixing is not fully suppressed, so additional bed height is required to compensate for it. Furthermore, additional bed height is required for the production of intermediate products in a consecutive reaction system to ensure the hydrogen is almost completely consumed. It should also be noted that the evaporation of solvent or reactant reduces the partial pressure of hydrogen, above all in the upper part of the reactor. This aspect necessitates additional catalyst. As a consequence the productivity of the reactor per unit of catalyst bed will only be a fraction of that for a reactor with cooling coils or catalyst in wall-cooled, small diameter tubes without evaporation. However, at the expense of productivity the reactor has a simple construction and does not need a hydrogen recycle compressor. We therefore expect it to be a very cheap, if not the cheapest, reactor.

\section{Notation}

$c_{i}$ concentration of component $i$ (mole $\mathrm{m}^{-3}$ )

$P \quad$ reactor pressure $(\mathrm{MPa})$

$q_{\mathrm{L}}$ liquid flow rate $\left(\mathrm{m}^{3} \mathrm{~s}^{-1}\right)$

$q$ gas flow rate $\left(\mathrm{nl} \mathrm{min} \mathrm{m}^{-1}\right)$

$T$ temperature $(\mathrm{K})$

$T_{\mathrm{o}}$ temperature of gas and liquid entering the reactor bed $(\mathrm{K})$

$u$ gas or liquid flow velocity $\left(\mathrm{m} \mathrm{s}^{-1}\right)$

$x_{i} \quad$ mole fraction of $i$-th component in liquid phase (-)

$X_{i} \quad$ relative mole fraction of $i$-th component

$z$ axial coordinate $(\mathrm{m})$
Greek letters

$\alpha_{\text {DAT }}$ hydrogen supply ratio (-)

$\eta \quad$ yield (-)

$\zeta_{j} \quad$ hydrogen conversion at $j$-th measurement

point. (-)

$\varphi \quad$ molar flow rate $\left(\mathrm{mol} \mathrm{s}{ }^{-1}\right)$

Subscripts and superscripts

in inlet

$\mathrm{G}$ gas

$\mathrm{N}_{2}$ nitrogen

0 initial

out outlet

$\mathrm{H}$ hydrogen

L liquid

Abbreviations

A 2,4-DNT (2,4-dinitrotoluene)

B 4-HA-2-NT (4-hydroxylamino-2-nitrotoluene)

C 4-A-2-NT (4-amino-2-nitrotoluene)

D 2-A-4-NT (2-amino-4-nitrotoluene)

E 2,4-DAT (2,4-diaminotoluene)

\section{Acknowledgements}

This work has been supported by the Netherlands Foundation for Chemical Research (SON). The following are gratefully acknowledged: Mr A. Pleiter for his technical support and advice, Prof. S. Wroński from the CPE Department of Warsaw Technical University for his interest and support, and also drir. M.H. Oyevaar, dr.ir. W. Wammes, dr.ir. H.J. Janssen en dr.ir. D. Stegeman, who also participated during the project.

\section{References}

[1] K.R. Westerterp, K.B. van Gelder, H.J. Janssen and M.H. Oycvaar, Devclopment of catalytic hydrogenation reactors for the fine chemicals industry, Chem. Eng. Sci., 43 (1988) $2229-$ 2236.

$[2]$ H.J. Janssen, A.J. Kruithof, G.J. Steghuis and K.R. Westerterp, Kinetics of the catalytic hydrogenation of 2,4-dinitrotoluene, 1. Experiments, reaction scheme and catalyst activity, Ind. Eng. Chem. Res., 29 (1990) 754-766.

[3] H.J. Janssen, A.J. Kruithof, G.J. Steghuis and K.R. Westerterp, Kinetics of the catalytic hydrogenation of 2,4-dinitrotoluene. 2. Modelling of the reaction rates and catalyst activity, Ind. Eng. Chem. Res., 29 (1990) 1822-1829.

[4] E.J. Molga and K.R. Westerterp, Kinetics of the hydrogenation of 2,4-dinitrotoluene over a palladium on alumina catalyst, Chem. Eng. Sci., 47 (7) (1992) 1733-1749. 
[5] K.R. Westerterp, H.J. Janssen and H.J. van der Kwast, The catalytic hydrogenation of 2,4-dinitrotoluene in a continuous stirred three-phase slurry reactor with an evaporating solvent, Chem. Eng. Sci., 47 (1992) 4179-4189.

[6] H.J. Janssen, H.J. Vos and K.R. Westerterp, A mathematical model for multiple hydrogeneration reactions in a continuous stirred three phase slurry reactor with an evaporating solvent, Chem. Eng. Sci., 47 (1992) 4191-4208.

[7] W.J.A. Wammes, S.J. Mechielsen and K.R. Westerterp, The transition between trickle flow and pulse flow in a cocurrent gas-liquid trickle-bed reactor at elevated pressures, Chem. Eng. Sci., 45 (1990) 3149-3158.

[8] W.J.A. Wammes and K.R. Westerterp, The influence of the reactor pressure on the hydrodynamics in a cocurrent gas-liquid trickle-bed reactor, Chem. Eng. Sci., 45 (1990) 2247-2254.

[9] W.J.A. Wammes, J. Middelkamp, W.J. Huisman, C.M. de Baas and K.R. Westerterp, Hydrodynamics in a cocurrent gas-liquid trickle bed at elevated pressures. Part I: gas-liquid interfacial areas, AIChE J., 37 (1991) 1849-1854.

[10] W.J.A. Wammes and K.R. Westerterp, Hydrodynamics in a pressurized cocurrent gas-liquid trickle-bed reactor, Chem. Eng. Technot., 14 (1991) 406-413.

[11] K.B. van Gelder, J.K. Damhof, P.J. Kroijenga and K.R. West- erterp, Three-phase packed bed reactor with an evaporating solvent - I. Experimental: the hydrogenation of 2,4,6-trinitrotoluene in methanol, Chem. Eng. Sci., 45 (1990) 3159-3170.

[12] K.B. van Gelder, P.C. Borman, R.E. Weenink and K.R. Westerterp, Three-phase packed bed reactor with an evaporating solvent - II. Modelling of the reactor, Chem. Eng. Sci., 45 (1990) 3171-3192.

[13] K.B. van Gelder and K.R. Westerterp, Residence time distribution and hold-up in a co-current upflow packed bed reactor at elevated pressure, Chem. Eng. Technol., 13 (1990) 27-40.

[14] M.H. Oyevaar, T. de la Rie, C.L. van der Sluijs and K.R. Westerterp, Interfacial areas and gas hold-ups in bubble columns and packed bubble columns at elevated pressures, Chem. Eng. Process., 26 (1989) 1-14.

[15] K.R. Westerterp, H.J. Fontein and F.P.H. van Beckum, Decoking of fixed-bed catalytic reactors, Chem. Eng. Technol,, 11 (1988) $367-375$.

[16] J.L. Turpin and R.L. Huntington, Prediction of pressure drop for two-phase, two-component flow in packed beds, AIChE J., 13 (1967) 1196-2001.

[17] M.Y. Saada, Assessment of interfacial area in cocurrent twophase flow in packed becis, Chem. Ind. Genie Chem., 105 (1972) $1415-1418$ 